\title{
Ramsey interferometry with generalized one-axis twisting echoes
}

\author{
Marius Schulte, Victor J. Martínez-Lahuerta, Maja S. Scharnagl, and Klemens Hammerer \\ Institut für Theoretische Physik und Institut für Gravitationsphysik (Albert-Einstein-Institut), Leibniz Universität Hannover, \\ Appelstraße 2, 30167 Hannover, Germany \\ 13. March 2020
}

We consider a large class of Ramsey interferometry protocols which are enhanced by squeezing and un-squeezing operations before and after a phase signal is imprinted on the collective spin of $N$ particles. We report an analytical optimization for any given particle number and strengths of (un-)squeezing. These results can be applied even when experimentally relevant decoherence processes during the squeezing and un-squeezing interactions are included. Noise between the two interactions is however not considered in this work. This provides a generalized characterization of squeezing echo protocols, recovering a number of known quantum metrological protocols as local sensitivity maxima, thereby proving their optimality. We discover a single new protocol. Its sensitivity enhancement relies on a double inversion of squeezing. In the general class of echo protocols, the newly found overun-twisting protocol is singled out due to its Heisenberg scaling even at strong collective dephasing.

\section{Introduction}

Atomic sensors are currently among the most accurate measuring apparatuses in metrology, for e.g. precision spectroscopy, magnetometry or frequency metrology. Therefore, they may help to detect minute effects which can be indications of new physics [1, 2]. For high-precision atomic sensors, a fundamental limitation is given by quantum fluctuations of the measurement. However, entanglement between atoms allows these fluctuations to be reduced below what is possible with uncorrelated probes [3]. In the optical domain, Marius Schulte: marius.schulte@itp.uni-hannover.de squeezed states have been injected into laser interferometers to enhance precision [4-6]. Likewise, significant successes followed in the generation and characterization of non-classical states in atomic physics [7]. However the biggest problems of these states are that strongly correlated systems often require demanding measurements and that increasing sensitivity typically comes at the cost of an increased susceptibility to imperfections. More detailed investigations, taking these effects into account, showed that the actual gain of such states can be significantly lower than in the ideal case [8-10]. Hence the question of practical quantum metrology protocols arises.

Recently, experiments in which simple but wellcontrolled interactions were used several times, in the form of an 'echo', achieved excellent results in a variety of precision measurements [1114]. The one-axis-twisting (OAT) interaction for effective two-level systems [15] allows a uniform description of many setups. It can be generated through cavity induced spin squeezing of cold atoms [7, 16], via laser or microwave driven quantum gates for trapped ions [17] and from spinchanging collisions in spinor Bose-Einstein condensates [7].

In our work we consider a large variational class of echo protocols based on OAT operations which, by construction, encompass a number of known protocols. In order to study which protocols give useful enhancements, we also include collective and individual dephasing during the OAT interactions. Noise during the probe time, i.e. the application of the phase, is, however, beyond the scope of this work. Related theoretical works include variational optimization algorithms [18] or inversion protocols using more general classes of spin-spin interactions which are referred to as interaction based readouts [19-24]. Here we obtain the maximal amplifications of signal-to- 
(a)

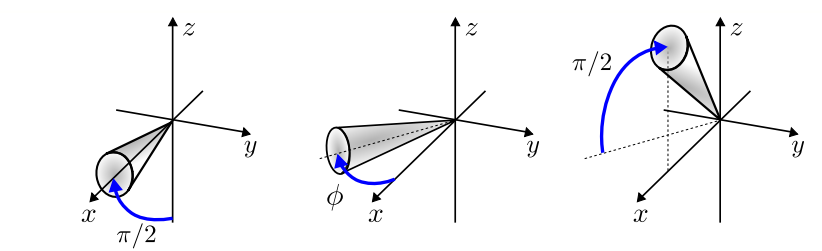

$|\downarrow\rangle \otimes N-\sqrt{\frac{\pi}{2}}$

(b)

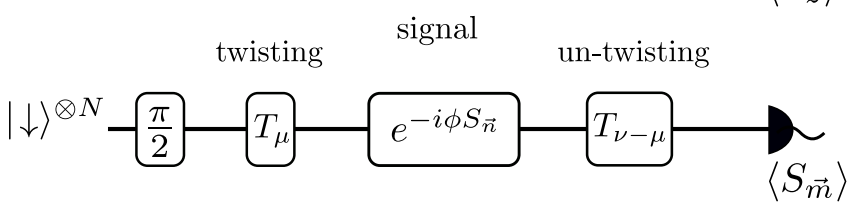

(c)

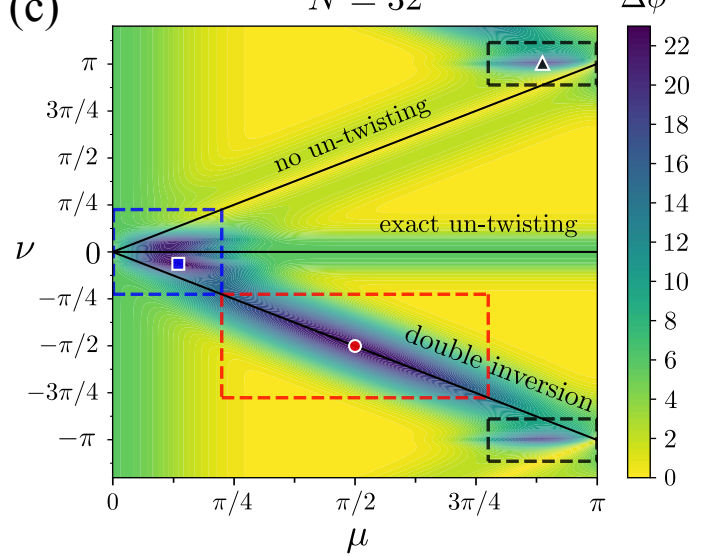

Figure 1: Generalized Ramsey protocols: (a) Conventional Ramsey measurement where first a phase $\phi$ is imprinted between two $\pi / 2$-pulses by rotating the state in the equatorial plane around the $z$-axis. Finally, the signal in phase is determined from measuring the spin component $S_{z}$. The cones visualize the mean spin direction and quantum fluctuations of the state during the protocol. (b) Generalized Ramsey protocols with additional one-axis twisting $T_{\mu}$ and un-twisting $T_{\nu-\mu}$ dynamics as well as arbitrary directions $\vec{n}, \vec{m}$ for the signal and the measurement respectively. Optimizing over these additional degrees of freedom allows to extract the best sensitivity, characterized by the initial squeezing strength $\mu$ and the inversion $\nu$ only. (c) Example of an optimized sensitivity landscape for the inverse phase variance $\Delta \phi^{-1}$ (around $\phi=0$ ) with $N=32$. The boxes (dashed lines) highlight three distinct types of protocols we identified. At small $\mu$ the 'squeezing protocols' (blue), and at large squeezing strength the 'GHZ protocols' (black), which are known in the literature. In between, at an unusual double inversion of squeezing for $\nu=-\mu$, we find a new class of 'over-un-twisting protocols' (red). Colored symbols denote the local maxima in each class.

noise ratio (SNR) by analytically optimizing geometric parameters, that is, signal and measurement directions. This allows to provide a complete overview and classification of our echo protocols in terms of the squeezing and un-squeezing strengths (at any level of dephasing and arbitrary $N)$. We identify one significant new scenario with a previously unused excess inversion. Such protocol types, which we refer to as 'overun-twisting' (OUT) protocols, are especially interesting as their sensitivity preserves the optimal Heisenberg scaling of entangled states, $\mathrm{SNR} \propto N$, independent of any collective dephasing during the OAT interactions.

\section{Generalized Ramsey protocols}

For a long time now, measurements of small phase shifts were established as the framework for precision experiments in atomic and optical physics. In this regard, many areas of atomic physics adapted Ramsey interrogation [25] due to the inherent reduction of systematic shifts. The conventional Ramsey protocol is depicted in Fig. 1(a). We consider $N$ two-level systems, described in terms of the collective spin operators $S_{x, y, z}=\frac{1}{2} \sum_{k=1}^{N} \sigma_{x, y, z}^{(k)}$ where $\sigma_{x, y, z}^{(k)}$ are the Pauli matrices for particle $k$. Starting from the product state $|\downarrow\rangle^{\otimes N}$ with all particles in the ground state, a rotation

$$
R_{\vec{n}}(\alpha)=e^{-i \alpha S_{\vec{n}}}
$$

around $\vec{n}=\vec{e}_{y}$ and with $\alpha=\pi / 2$ generates the coherent spin state $|\mathrm{x}\rangle=\left[\frac{|\downarrow\rangle+|\uparrow\rangle}{\sqrt{2}}\right]^{\otimes N}$, polarized in $x$-direction. (We use the notation $S_{\vec{n}}=\vec{n} \cdot \vec{S}$ ). In the following interaction period, a small phase signal $\phi$ is imprinted by rotating around the $z$ axis. A second $\pi / 2$ rotation, with $\vec{n}=\vec{e}_{x}$, allows to infer $\phi$ from the measurement result of $S_{z}$.

Motivated by the conventional Ramsey scheme, we now consider the generalized echo protocols shown schematically in Fig 1(b). Starting again from the coherent spin state $|\mathrm{x}\rangle$ after the first $\pi / 2$ pulse, a squeezed spin state is generated via the non-linear OAT interaction $T_{\mu}=e^{-i \frac{\mu}{2} S_{z}^{2}}$, with strength $\mu$. The necessary Hamiltonian $\propto S_{z}^{2}$ can be engineered in a variety of metrologically relevant systems [7, 17]. For small squeezing strength, $\mu<4 / \sqrt{N}$, the generated entanglement is reflected by reduced fluctuations of the mean spin $[7,15]$. With increasing $\mu$ greater levels of multi-particle entanglement are generated. At $\mu=\pi$ rotated versions of the $N$-particle correlated Greenberger-Horne-Zeilinger (GHZ) state 
$\left[|\downarrow\rangle^{\otimes N}+|\uparrow\rangle^{\otimes N}\right] / \sqrt{2}$, aligned along the $x$ axis if $N$ is even or along the $y$ axis if $N$ is odd, are created and the dynamics reverses afterwards [7]. For the following signal imprint we now allow the more general case of a rotation of the collective spin around an arbitrary axis $\vec{n}$. Note that a physical rotation around the $z$-axis may be converted to a rotation around $\vec{n}$ by appropriate single qubit rotations $R$ (in the form of Eq. (1)) before and after the phase imprint such that $R_{\vec{n}}(\phi)=e^{-i \phi S_{\vec{n}}}=R^{-1} e^{-i \phi S_{z}} R$. Prior to the measurement we allow for another OAT interaction with strength $\nu-\mu$. With this definition, $\nu$ describes the deviation from an exact inversion of the initial OAT. This choice is based on an appearing symmetry around exact echo protocols at $\nu=0$, c.f. Fig. 1(c). We assume inversion of OAT is possible by reverting the sign of the interaction strength, as already demonstrated for cold atoms [11], spinor Bose-Einstein condensates [13] and trapped ions [12, 26]. At the end of our protocols the collective spin $S_{\vec{m}}$ in an arbitrary direction $\vec{m}$ is measured. Again, this can be implemented with a measurement of $S_{z}$ by preceding an appropriate rotation of the collective spin. Overall, the generalized echo protocols have a measurement signal

$$
\left\langle S_{\vec{m}}\right\rangle(\phi)=\left\langle\mathrm{x}\left|T_{\mu}^{\dagger} R_{\vec{n}}^{\dagger}(\phi) T_{\nu-\mu}^{\dagger} S_{\vec{m}} T_{\nu-\mu} R_{\vec{n}}(\phi) T_{\mu}\right| \mathrm{x}\right\rangle
$$

characterized by parameters $\mu, \nu$ for squeezing and un-squeezing and directions $\vec{n}, \vec{m}$ for signal and measurement.

We highlight that the generalized echo protocols include some common squeezing protocols as limiting cases: For $\nu=\mu$, with no un-twisting, we find standard Ramsey interrogation with a spin squeezed initial state [27]. In this case the SNR is enhanced by reducing projection noise at a constant signal. More recently, protocols with exact inversion, i.e. $\nu=0$, were suggested for application in quantum metrology [28-30]. There, amplification of the signal at constant measurement noise occurs.

\section{Geometric optimization}

In the following we quantify metrological gain by the inferred phase deviation

$$
\Delta \phi(\mu, \nu, \vec{n}, \vec{m})=\left.\Delta S_{\vec{m}}\right|_{\phi=0} /\left|\frac{\partial\left\langle S_{\vec{m}}\right\rangle}{\partial \phi}\right|_{\phi=0} \mid
$$

around the working point $\phi=0$. This is a measure of sensitivity which describes enhancements in atomic sensors limited by quantum projection noise. We will now show that the essential optimization with respect to the signal and measurement directions can be solved analytically. The method described in the following corresponds exactly to the method first described by Gessner et al. in [31]. While in that case the authors were able to systematically optimize measurement operators, we use the method in our work to find the optimal geometric factors for generalized echo protocols. It is interesting to note that only by this means the metrological gain of strongly entangled states, such as non-Gaussian states, could be shown in both works.

First, we re-express the two contributions, signal and noise, separately. For the signal strength we find

$$
\left|\frac{\partial\left\langle S_{\vec{m}}\right\rangle}{\partial \phi}\right|_{\phi=0} \mid=\vec{n}^{T} M \vec{m}
$$

with $M_{k l}=i\left\langle\left[S_{k}(\mu), S_{l}(\nu)\right]\right\rangle_{\left.\right|_{\phi=0}}$ where we denoted transformed spin operators by $S_{\vec{n}}(\mu)=$ $T_{\mu}^{\dagger} S_{\vec{n}} T_{\mu}$. Likewise, the measurement variance can be expressed as

$$
\Delta S_{\left.\vec{m}\right|_{\phi=0} ^{2}}^{2}=\left\langle S_{\vec{m}}^{2}(\nu)-\left\langle S_{\vec{m}}(\nu)\right\rangle^{2}\right\rangle_{\left.\right|_{\phi=0}}=\vec{m}^{T} Q \vec{m}
$$

with $Q_{k l}=\left\langle S_{k}(\nu) S_{l}(\nu)-\left\langle S_{k}(\nu)\right\rangle\left\langle S_{l}(\nu)\right\rangle\right\rangle_{\left.\right|_{\phi=0}}$. The matrices $M$ and $Q$ can be obtained analytically (see appendix A).

For sensitivity optimization we aim to maximize the inverse phase deviation, i.e. SNR, which is expressed as

$$
\Delta \phi^{-1}=\frac{\vec{n}^{T} M \vec{m}}{\sqrt{\vec{m}^{T} Q \vec{m}}} .
$$

Because $Q$ is a positive semi-definite spin covariance matrix, we can define the vector $\vec{v}=$ $Q^{1 / 2} \vec{m}$ with $\|\vec{v}\|=\sqrt{\vec{m}^{T} Q \vec{m}}$ and correspondingly $\vec{m}=Q^{-1 / 2} \vec{v}$. Note that $Q$ is singular for $\nu=0$ only. In this case, the optimization of rotation and measurement directions can be restricted to the plane perpendicular to the initial spin polarization. With the unit vector $\vec{u}=\vec{v} /\|\vec{v}\|$ the sensitivity is

$$
\Delta \phi^{-1}=\vec{n}^{T} M Q^{-1 / 2} \vec{u}
$$

This can be optimized over all signal directions $\vec{n}$ and measurement directions $\vec{m}$ by a singular value decomposition for $M Q^{-1 / 2}$. After this step 
the sensitivity depends exclusively on the initial squeezing strength $\mu$ and excess inversion $\nu$. The ideal directions for signal and measurement, at each point $(\mu, \nu)$, can be inferred from two orthogonal matrices which are determined by the singular value decomposition. An example landscape of the optimal SNR, i.e. maximal singular value,

$$
\Delta \phi^{-1}(\mu, \nu)=\max _{\vec{n}, \vec{m}} \Delta \phi^{-1}(\mu, \nu, \vec{n}, \vec{m})
$$

is shown in Fig. 1(c). We point out that the relatively small particle number, $N=32$, there is motivated to easily highlight important features of the landscape. With the analytic expressions, computational time is independent of $N$ and we show landscapes for larger particle numbers in the appendix, Fig. 6.

In view of Fig. 1(c) mainly three separate regions exist in which amplified SNR is achieved: (i) The first region (blue box) includes protocols with small squeezing strengths $|\mu|,|\nu| \lesssim 4 / \sqrt{N}$. We refer to such cases as 'squeezing protocols' because this is the only region that contains initial states exhibiting reduced spin fluctuations while still maintaining significant polarization. Note however that this usual intuition is no longer exclusive for all states in this class. Already at $\mu \gtrsim 2 / \sqrt{N}$ the states generated by OAT enter the regime of so called oversqueezed states, which wrap around the Bloch sphere. From this point on spin squeezing is lost, i.e. $\xi>1$, according to the Wineland squeezing parameter $\xi[32,33]$. This is connected to our figure of merit without any echo $(\nu=\mu)$ by $\xi=N \Delta \phi^{2}(\mu, \mu)$. While the original classification based on the squeezing parameter $\xi$ fails with the application of echoes, we find that the characteristic scaling $\sim 1 / \sqrt{N}$ with particle number remains valid for the generalized protocols. Note that the failure of this argument is also visible in the landscapes we show. Compare e.g. the position of the maximum along the $\nu=\mu$ line with the local maximum over all squeezing protocols (blue square) in Fig. 1(c). The additional factor of two in the condition $|\mu|,|\nu| \lesssim 4 / \sqrt{N}$ was introduce here to place the cutoff close to the minimum that lies between the local maximum at small squeezing strength and the broad maximum around $\mu=\pi / 2$, cf. Fig.2(b).

As a special case of 'squeezing protocols' we recover the classic Ramsey protocols with squeezed initial states and no un-squeezing $(\nu=\mu)$ along with their known optimal signal and measurement directions [15]. We also find optimized exact echo protocols, on the horizontal line at $\nu=0$, for initial squeezing strengths $\mu \sim 2 / \sqrt{N}$. The optimal directions converge to $\vec{n}=\vec{e}_{y}$ and $\vec{m}=\vec{e}_{y}$ for $N \gg 1$. This proves optimality of the choices of signals and measurements made by Davis et al. [28]. Interestingly, we find that within the class of squeezing protocols the local maximum in sensitivity is reached at values of $\nu$ which do not correspond to either of these known protocols. (ii) The second region (black box) exhibiting enhanced sensitivity exists at large squeezing strength, $\pi-4 / \sqrt{N} \leq \mu \leq \pi$, which contains highly entangled states close to the GHZ state, so that we refer to these as 'GHZ protocols'. Note that the enhancement of GHZ states is ideally obtained with parity measurements. Recently, approaches using the measurement of spin projections and an additional squeezing interaction were discussed by Leibfried et al. [12, 34]. (iii) Finally, we find exactly one more region (red box, with $4 / \sqrt{N}<\mu<\pi-4 / \sqrt{N}$ ) corresponding to a new type of protocols that are characterized by a double inversion of the OAT, at $\nu=-\mu$. We refer to these as 'over-un-twisting' (OUT) protocols. The enhancing mechanism there is conceptually different from the squeezing protocols and is discussed further below. The initial states in this class are regarded as oversqueezed or nonGaussian states [35]. So far the entanglement was first captured by the quantum Fisher information [36] or only in terms of nonlinear squeezing parameters [31].

\section{Dephasing noise}

To see which protocols actually correspond to a robust enhancement, we now add dephasing during the OAT. In the presence of collective dephasing, at a rate $\gamma_{C}>0$, the dynamics of the system will be governed by the master equation

$$
\dot{\rho}=-i[H, \rho]+\gamma_{C} \mathcal{L}_{C}[\rho]
$$

with $H=\chi S_{z}^{2}$ and $\mathcal{L}_{C}[\rho]=S_{z} \rho S_{z}-\frac{1}{2} S_{z}^{2} \rho-\frac{1}{2} \rho S_{z}^{2}$. The noise strength is quantified by the dimensionless parameter $\sigma=\left|\gamma_{C}\right| /|\chi|$. In cavity induced squeezing of atoms such dephasing occurs as fluctuations in the phase of the collective spin due to photon shot noise [28]. For quantum gates with 

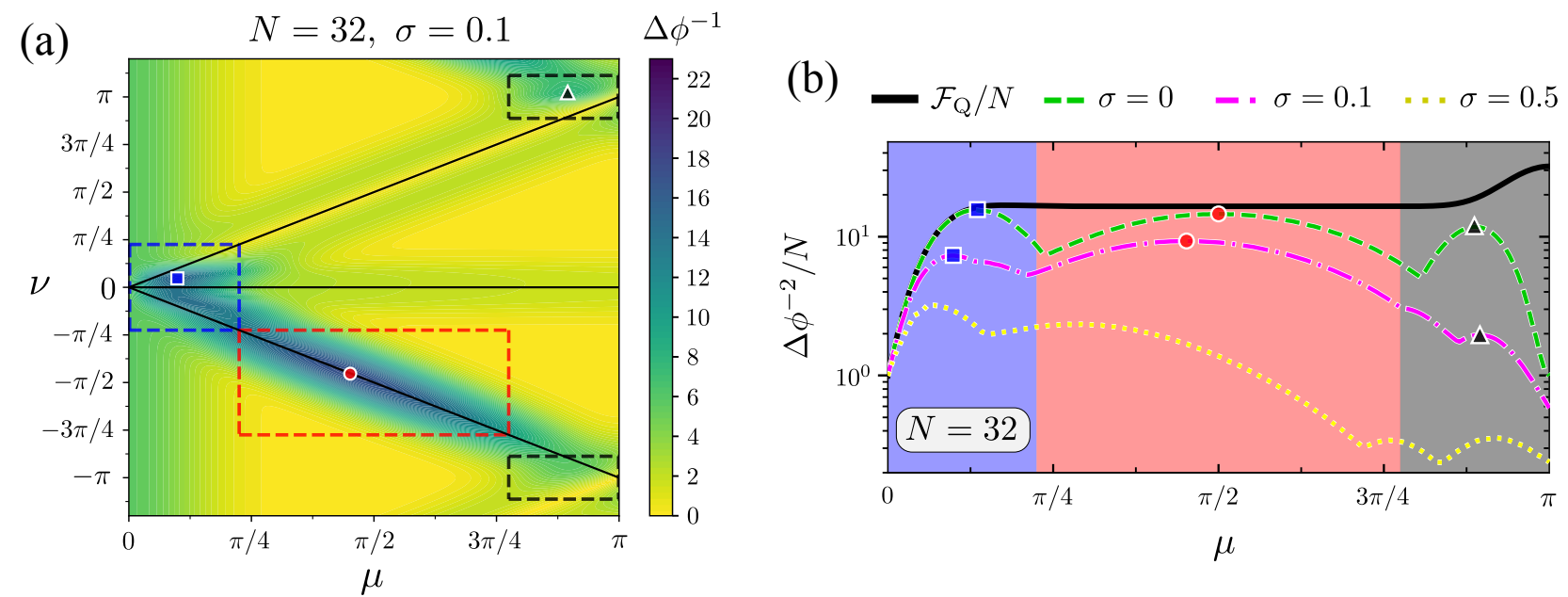

Figure 2: Sensitivity with dephasing: (a) Sensitivity $\Delta \phi^{-1}$ for $N=32$ with $\sigma=0.1$ collective dephasing noise during the state preparation and inversion. The overall structure of generalized echo protocols remains intact. As compared to Fig. 1(c), changes in the positions of optimal protocols (symbols) as well as an overall reduction in sensitivity can be observed. (b) SNR with optimized inversion, $\max _{\nu} \Delta \phi^{-2}(\mu, \nu) / N$, for different levels of collective dephasing noise $\sigma=0,0.1,0.5$. Colored areas mark the squeezing, OUT and GHZ protocol types. The black line is the scaled quantum Fisher information $\mathcal{F}_{Q} / N$ of the ideal initial squeezed state, i.e. $\sigma=0$. We find three peaks corresponding to the optimal squeezing, over-un-twisting and $\mathrm{GHZ}$ protocols. With increasing particle number, only the over-un-twisting protocols are robust to small collective dephasing with a sensitivity close to the fundamental quantum Fisher information limit. Symbols on the green dashed line represent the optimal protocols of Fig. 1(c) while symbols on the magenta dash-dotted line correspond to part (a).

trapped ions, dephasing occurs through random variations of the transition frequency from stray fields or from frequency noise of the driving fields. When uniform over the extend of the ion string, both result in collective dephasing [37, 38]. With spinor Bose-Einstein condensates, collective dephasing may again arise from magnetic field fluctuations [7].

The geometrical optimization with respect to $\vec{n}$ and $\vec{m}$ can again be performed analytically, see appendix B. Figure 2(a) shows the sensitivity including collective dephasing with $\sigma=0.1$. Compared to the ideal case, shown in Fig. 1(c), we see that any enhancement of the GHZ protocols is strongly suppressed by the noise. Furthermore, within the squeezing protocols a bias towards $\nu \approx \mu$ is developing as protocols with reduced additional inversion suffer less from dephasing. Surprisingly, the large maximum of OUT protocols remains only weakly affected by preparation noise and still offers large enhancement. To emphasize this, Fig. 2(b) displays the measurement optimized sensitivity $\max _{\nu}\left(\Delta \phi^{-2}(\mu, \nu) / N\right)$ as a function of the initial squeezing at various levels of dephasing. Performing the optimization over all measurements within our protocols also allows to compare the obtained SNR to funda-
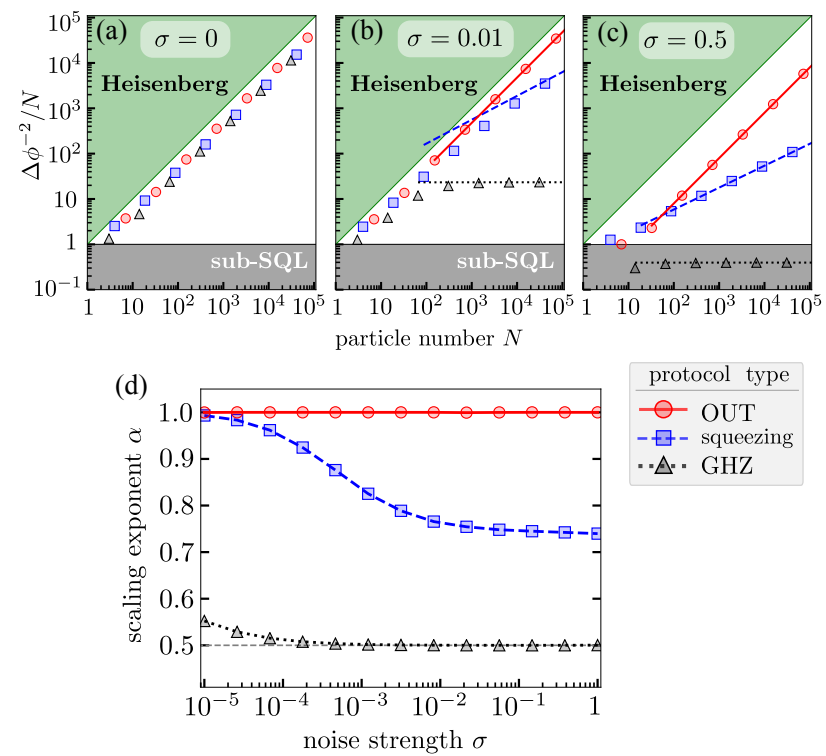

Figure 3: Scaling of sensitivity with particle number: (a)-(c) Scaling of the sensitivity under collective dephasing with $\sigma=0.01,0.1,0.5$ for squeezing (blue), OUT (red) and GHZ (black) protocols. The green region shows sensitivity beyond the Heisenberg limit, $\Delta \phi^{-2}>N^{2}$, and the grey region sensitivity below the standard quantum limit, $\Delta \phi^{-2}<N$. Lines are fits of the local optima to asymptotic scalings $\Delta \phi^{-1}=c N^{\alpha}$ at large $N$. Note that a flat line thus still means improvement with $N$, however only at the classical scaling $\propto N$. (d) Scaling exponent $\alpha$ as a function of noise strength for the three protocol types identified above. 
mental limits of quantum metrology. Most notably, the quantum Cramér-Rao bound implies $\max _{\nu} \Delta \phi^{-2}(\mu, \nu) \leq \mathcal{F}_{Q}$. The upper limit $\mathcal{F}_{Q}$ is the quantum Fisher information, which quantifies the maximum information about the phase $\phi$ that can possibly be inferred from the initial squeezed state under any rotation $S_{\vec{n}}$. The quantum Fisher information thereby includes an optimization over all measurements, including weak measurements, individual operations on each particle, parity and others, which go beyond what is possible with the resources considered here. Based on [36] we show the quantum Fisher information limit (black line) in Fig. 2(b) in comparison to the SNR. As a function of the squeezing strength, $\mathcal{F}_{\mathrm{Q}}$ increases from the standard quantum limit $\mathcal{F}_{\mathrm{Q}}=N$ of uncorrelated particles at $\mu=0$ up to the Heisenberg limit $\mathcal{F}_{\mathrm{Q}}=N^{2}$ at $\mu=\pi$. Even though the quantum Fisher information constitutes a true extension over the capabilities of the protocols considered here, we nevertheless find that the OUT protocols actually reach the quantum Fisher information bound with increasing $N$. This feature persists for small dephasing as well. The only other case where this holds true is for $\mu \ll 1$. However, at extreme levels of noise also the OUT protocols fall short compared to the quantum Fisher information limit.

Due to the exact optimization introduced above we are also able to efficiently examine the influenced of dephasing on the particle number scaling of the sensitivity. Figure 3(a)-(c) show the scalings for $\sigma=0,0.01,0.5$. Symbols mark the best sensitivity within each protocol type while lines show numerical fits to an asymptotic scaling $\Delta \phi^{-1}=c N^{\alpha}$ with fitting parameters $c, \alpha$. The green region reflects sensitivity beyond the Heisenberg limit $\Delta \phi^{-2}>N^{2}$ and the grey region sensitivity below the standard quantum limit $\Delta \phi^{-2}<N$. We find that, remarkably, the OUT protocols always exhibit Heisenberg scaling, $\Delta \phi^{-1} \propto N$, independent of the dephasing. On the other hand the GHZ protocols quickly drop to the classic scaling, showcasing their increased susceptibility in this regard. The dependence of the exponent $\alpha$ on the noise strength is shown in Fig. 3(d), highlighting the characteristic differences regarding the influence of collective dephasing. Although the squeezing protocols have a reduced scaling exponent compared to OUT protocols, they may still be the overall best proto-
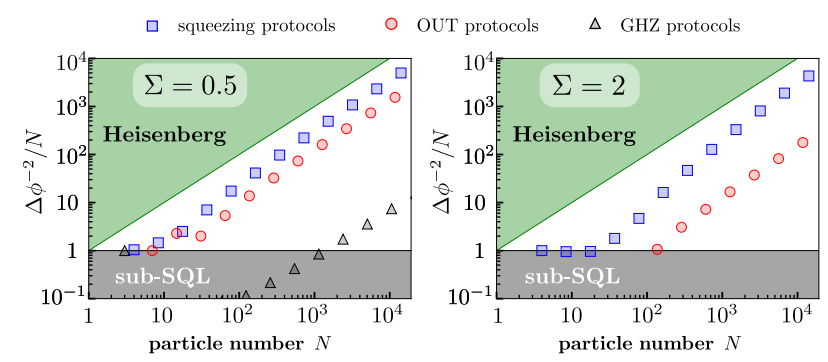

Figure 4: Effects of individual dephasing: Sensitivity versus particle number for individual dephasing during the OAT interactions with strength $\Sigma=0.5$ and extreme dephasing at $\Sigma=2$.

col when limited to small ensembles and larger dephasing, cf. Fig. 3(c). Our findings also show that initially the scaling may deviate significantly from the asymptotic case, even up to ensembles of considerable size.

In addition to collective dephasing we also study individual dephasing during the OAT interactions. Compared to Eq. (9), the master equation is $\dot{\rho}=-i[H, \rho]+\gamma_{I} \mathcal{L}_{I}[\rho]$, where $\mathcal{L}_{I}[\rho]=$ $\sum_{k=1}^{N} \sigma_{z}^{k} \rho \sigma_{z}^{k}-\rho$. This describes individual but symmetric dephasing of each atom at a rate $\gamma_{I}>$ 0 and we likewise define $\Sigma=\left|\gamma_{I}\right| /|\chi|$. It turns out that individual dephasing results in a less stringent restriction on sensitivity than collective dephasing. The sensitivity for all protocol types scales asymptotically linearly in $N$, independent of the noise strength, as shown in Fig. 4.

\section{Over-un-twisting enhancement}

In this last section we give an explanation of the mechanism underlying the OUT protocols. To visualize the sensitivity enhancement we split the protocols into a state evolution and an effective measurement part. As shown in the schematic of Fig. 5 we group one half of the double inversion to the state evolution and the other half to the measurement. Now for the optimal OUT protocol, initially a superposition of four coherent spin states along the equator of the collective Bloch sphere is generated by $T_{\mu}$ with $\mu=\frac{\pi}{2}$ [39], as shown by the Wigner function [40] in the top row of Fig. 5.

A small rotation around the $y$-axis perturbs the following exact inversion of OAT in such a way that interference patterns remain on the sides of a large polarization contribution in $x$-direction. The absolute values of these patterns increase 


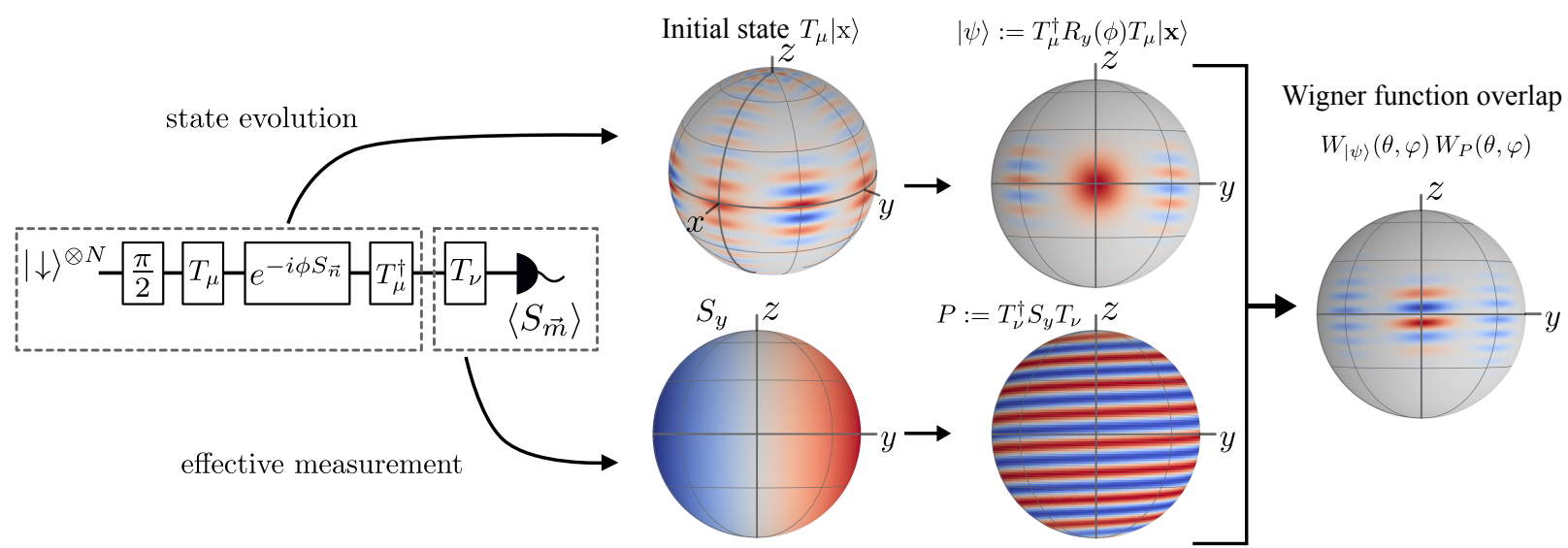

Figure 5: Illustration of over-un-twisting protocols: Wigner function representation for the optimal over-untwisting protocol at $\mu=\frac{\pi}{2}, \nu=-\frac{\pi}{2}$ and with $N=32$. Half the OAT inversion is assigned to the state evolution, the other half to the measurement operator. State evolution (top): Starting from the superposition of four coherent states along the equator, a small rotation around $y$ (here $\phi=-0.02$ ) followed by exact OAT inversion, leads to the state $|\psi\rangle$ with large polarization in $x$-direction and residual interference patterns due to the disturbance of the rotation. Effective measurement (bottom): OAT of the optimal measurement direction $\approx S_{y}$ creates dense sequences of positive and negative values in its Wigner function. These match exactly the pattern on $|\psi\rangle$, so that in the overlap of the two Wigner functions the interference patterns are either all positive or all negative, depending on the sign of $\phi$. Integrating the overlap over the sphere results in the measurement signal $\left\langle S_{y}\right\rangle$. Larger values of $N$ have narrower spacing of the interference patterns with increased maximal and minimal values of the Wigner function, thus giving an enhanced signal.

for larger rotation angles $\phi$ or with increasing $N$ when keeping $\phi \ll 1$ fixed. The signs of the interference patterns change only when rotating in the opposite direction, i.e. $R_{y}(-\phi)$ instead of $R_{y}(\phi)$. The second part of the OUT protocols can be viewed as a transformation of the applied measurement. The bottom row in Fig. 5 shows that the twisting dynamics on $S_{y}$ leads to a Wigner function for the operator $P:=$ $T_{\pi / 2}^{\dagger} S_{y} T_{\pi / 2}$ with rapid sign changes, wrapping around the Bloch sphere. Note that the density of patterns increases in the same way with $N$ for both the state $|\psi\rangle$ and the measurement operator $P$. So importantly the interference patterns of the two Wigner functions match exactly. It is interesting to note that the Wigner functions show similarities to a Moiré pattern as well as Gottesman-Kitaev-Preskill states [41, 42]. Due to the matching Wigner functions, in the product all oscillations contribute with either a positive or a negative sign, depending on the sign of the rotation. The mean $\left\langle S_{y}\right\rangle=$ $\int_{0}^{\pi} \mathrm{d} \theta \int_{0}^{2 \pi} \mathrm{d} \varphi \sin (\theta) W_{|\psi\rangle}(\theta, \varphi) W_{P}(\theta, \varphi)$ then corresponds to the integral over the sphere for the product of the Wigner functions [40, 43]. This shows $N$ times faster oscillations, compared to uncorrelated atoms, for even $N$. For odd $N$ the signal has a sharp initial incline before vanish- ing after a few oscillations (see Fig. 7 in the appendix). Although this distinction will be relevant for larger phases $\phi$, both the figure of merit in Eq. (3) and the optimal signal and measurement directions remain a continuous function of $N$. Thus, for the OUT protocols no additional information about the particle number is necessary. This is a consequence of the fact that we study and optimize the local sensitivity around $\phi=0$. Beyond the local sensitivity the mean information obtained per estimate can be calculated following [44]. We find that the information is similar to what is achieved with coherent spin states, in stark contrast to GHZ states which return only a single bit of information. The fact that we find an increased information gain, as well as a large local sensitivity is a compelling property of this novel class of protocols.

\section{Summary and Outlook}

In conclusion, we presented an analytic theory for the geometric optimization of generalized echo protocols at any particle number and dephasing. The same optimization was already treated in [31] by Gessner, Smerzi and Pezzè. Using these results, we are able to give a comprehensive overview and characterization of the vari- 
ational class of echo protocols in terms of the (un-)squeezing strengths. We find that only one new protocol exists. This protocol stands out as it exhibits Heisenberg scaling in the sensitivity even for strong dephasing during the OAT interaction. Remarkably, the effectively nonlinear readout performs almost as well as the quantum Fisher information in this case. Our results provide a route towards quantum enhanced measurements away from the typical squeezing regime or GHZ states with the measurement of spin projections only. Beyond the scope of this paper, non-ideal signal and measurement directions or the impact of other imperfections such as noise during the phase imprint can be studied. The former is not expected to reduce sensitivity much for directions close to the optimal ones due to the predominant contribution of the largest singular value. The exact effect of the latter often depends on a number of other factors and requires additional modelling. For example a more precise modelling of the physical system at hand, the duration of the interrogation and the type and strength of the noise. It is known that, beyond some critical ensemble size, noise during the signal acquisition reduces the scaling of quantum metrological amplification to the standard (classical) scaling of independent particles [9, 10]. This behavior is also expected for our protocols, most notably for the highly entangled states beyond the squeezing regime. In most cases, however, it is the absolute performance at a given $N$ which matters and not the scaling. We therefore believe that the tradeoff between quantum projection noise and technical noise, specific to each sensor, must be studied to understand in which cases entanglement is actually useful for metrological improvements [45].

\section{Acknowledgments}

We would like to thank C. Klempt for helpful discussions and J.P. Home for commenting on the possible connection to GKP protocols. This work was funded by the Deutsche Forschungsgemeinschaft (DFG, German Research Foundation) under Germany's Excellence Strategy - EXC-2123 QuantumFrontiers - 390837967 and CRC 1227 'DQ-mat' project A06. The publication of this article was funded by the Open Access Fund of Leibniz Universität Hannover.

\section{References}

[1] A. D. Ludlow, M. M. Boyd, J. Ye, E. Peik, and P. O. Schmidt. Optical atomic clocks. Rev. Mod. Phys., 87:637-701, Jun 2015. DOI: https://doi.org/10.1103/RevModPhys.87.637.

[2] M. S. Safronova, D. Budker, D. DeMille, D. F. J. Kimball, A. Derevianko, and C. W. Clark. Search for new physics with atoms and molecules. Rev. Mod. Phys., 90:025008, Jun 2018. DOI: https://doi.org/10.1103/RevModPhys.90.025008.

[3] V. Giovannetti, S. Lloyd, and L. Maccone. Quantum metrology. Phys. Rev. Lett., 96:010401, Jan 2006. DOI: https://doi.org/10.1103/PhysRevLett.96.010401.

[4] L. Barsotti, J. Harms, and R. Schnabel. Squeezed vacuum states of light for gravitational wave detectors. Reports on Progress in Physics, 82(1):016905, Dec 2018. DOI: https://doi.org/10.1088/1361-6633/aab906.

[5] M. Tse et al. Quantum-enhanced advanced LIGO detectors in the era of gravitational-wave astronomy. Phys. Rev. Lett., 123:231107, Dec 2019. DOI: https://doi.org/10.1103/PhysRevLett.123.231107.

[6] F. Acernese et al. Increasing the astrophysical reach of the advanced Virgo detector via the application of squeezed vacuum states of light. Phys. Rev. Lett., 123:231108, Dec 2019. DOI: https://doi.org/10.1103/PhysRevLett.123.231108.

[7] L. Pezzè, A. Smerzi, M. K. Oberthaler, R. Schmied, and P. Treutlein. Quantum metrology with nonclassical states of atomic ensembles. Rev. Mod. Phys., 90:035005, Sep 2018. DOI: https://doi.org/10.1103/RevModPhys.90.035005.

[8] S. F. Huelga, C. Macchiavello, T. Pellizzari, A. K. Ekert, M. B. Plenio, and J. I. Cirac. Improvement of frequency standards with quantum entanglement. Phys. Rev. Lett., 79:3865-3868, Nov 1997. DOI: https://doi.org/10.1103/PhysRevLett.79.3865.

[9] B. M. Escher, R. L. de Matos Filho, and L. Davidovich. General framework for estimating the ultimate precision limit in noisy quantum-enhanced metrology. $\mathrm{Na}$ ture Physics, 7(5):406-411, Mar 2011. DOI: https://doi.org/10.1038/nphys1958. 
[10] R. Demkowicz-Dobrzański, J. Kołodyński, and M. Guţă. The elusive Heisenberg limit in quantum-enhanced metrology. Nature Communications, 3(1):1063, Jan 2012. DOI: https://doi.org/10.1038/ncomms2067.

[11] O. Hosten, R. Krishnakumar, N. J. Engelsen, and M. A. Kasevich. Quantum phase magnification. Science, 352(6293):1552-1555, Jun 2016. DOI: https://doi.org/10.1126/science.aaf3397.

[12] D. Leibfried, M. D. Barrett, T. Schaetz, J. Britton, J. Chiaverini, W. M. Itano, J. D. Jost, C. Langer, and D. J. Wineland. Toward Heisenberg-limited spectroscopy with multiparticle entangled states. Science, 304(5676):1476-1478, Jun 2004. DOI: https://doi.org/10.1126/science.1097576.

[13] D. Linnemann, H. Strobel, W. Muessel, J. Schulz, R. J. Lewis-Swan, K. V. Kheruntsyan, and M. K. Oberthaler. Quantum-enhanced sensing based on time reversal of nonlinear dynamics. Phys. Rev. Lett., 117:013001, Jun 2016. DOI: https://doi.org/10.1103/PhysRevLett.117.013001.

[14] S. C. Burd, R. Srinivas, J. J. Bollinger, A. C. Wilson, D. J. Wineland, D. Leibfried, D. H. Slichter, and D. T. C. Allcock. Quantum amplification of mechanical oscillator motion. Science, 364 (6446):1163-1165, Jun 2019. DOI: https://doi.org/10.1126/science.aaw2884.

[15] M. Kitagawa and M. Ueda. Squeezed spin states. Phys. Rev. A, 47: 5138-5143, Jun $1993 . \quad$ DOI: https://doi.org/10.1103/PhysRevA.47.5138.

[16] M. H. Schleier-Smith, I. D. Leroux, and V. Vuletić. States of an ensemble of two-level atoms with reduced quantum uncertainty. Phys. Rev. Lett., 104:073604, Feb 2010. DOI: https://doi.org/10.1103/PhysRevLett.104.073604.

[17] R. Blatt and D. Wineland. Entangled states of trapped atomic ions. Nature, 453(7198):1008-1015, Jun 2008. DOI: https://doi.org/10.1038/nature07125.

[18] R. Kaubruegger, P. Silvi, C. Kokail, R. van Bijnen, A. M. Rey, J. Ye, A. M. Kaufman, and P. Zoller. Variational spin-squeezing algorithms on programmable quantum sensors. Phys. Rev. Lett., 123:260505, Dec 2019. DOI: https://doi.org/10.1103/PhysRevLett.123.26050[28] E. Davis, G. Bentsen, and M. Schleier-
[19] T. Macrì, A. Smerzi, and L. Pezzè. Loschmidt echo for quantum metrology. Phys. Rev. A, 94:010102, Jul 2016. DOI: https://doi.org/10.1103/PhysRevA.94.010102.

[20] S. A. Haine. Using interactionbased readouts to approach the ultimate limit of detection-noise robustness for quantum-enhanced metrology in collective spin systems. Phys. Rev. A, 98:030303, Sep 2018. DOI: https://doi.org/10.1103/PhysRevA.98.030303.

[21] S. S. Mirkhalaf, S. P. Nolan, and S. A. Haine. Robustifying twist-and-turn entanglement with interaction-based readout. Phys. Rev. A, 97:053618, May 2018. DOI: https://doi.org/10.1103/PhysRevA.97.053618.

[22] F. Anders, L. Pezzè, A. Smerzi, and C. Klempt. Phase magnification by two-axis countertwisting for detectionnoise robust interferometry. Phys. Rev. A, 97:043813, Apr 2018. DOI: https://doi.org/10.1103/PhysRevA.97.043813.

[23] J. Huang, M. Zhuang, B. Lu, Y. Ke, and C. Lee. Achieving Heisenberglimited metrology with spin cat states via interaction-based readout. Phys. Rev. A, 98:012129, Jul 2018. DOI: https://doi.org/10.1103/PhysRevA.98.012129.

[24] A. Niezgoda, D. Kajtoch, J. Dziekańska, and E. Witkowska. Optimal quantum interferometry robust to detection noise using spin-1 atomic condensates. New Journal of Physics, 21(9):093037, Sep 2019. DOI: https://doi.org/10.1088/1367-2630/ab4099.

[25] N. F. Ramsey. A molecular beam resonance method with separated oscillating fields. Phys. Rev., 78:695-699, Jun 1950. DOI: https://doi.org/10.1103/PhysRev.78.695.

[26] M. Gärttner, J. G. Bohnet, A. Safavi-Naini, M. L. Wall, J. J. Bollinger, and A. M. Rey. Measuring out-of-time-order correlations and multiple quantum spectra in a trapped-ion quantum magnet. Nature Physics, 13(8):781-786, May 2017. DOI: https://doi.org/10.1038/nphys4119.

[27] A. André, A. S. Sørensen, and M. D. Lukin. Stability of atomic clocks based on entangled atoms. Phys. Rev. Lett., 92:230801, Jun 2004. DOI: https://doi.org/10.1103/PhysRevLett.92.230801. 
Smith. Approaching the Heisenberg limit without single-particle detection. Phys. Rev. Lett., 116:053601, Feb 2016. DOI: https://doi.org/10.1103/PhysRevLett.116.05360138

[29] F. Fröwis, P. Sekatski, and W. Dür. Detecting large quantum Fisher information with finite measurement precision. Phys. Rev. Lett., 116:090801, Mar 2016. DOI: https://doi.org/10.1103/PhysRevLett.116.09080139

[30] S. P. Nolan, S. S. Szigeti, and S. A. Haine. Optimal and robust quantum metrology using interaction-based readouts. Phys. Rev. Lett., 119:193601, Nov 2017. DOI: https://doi.org/10.1103/PhysRevLett.119.193601.

[31] M. Gessner, A. Smerzi, and L. Pezzè. Metrological nonlinear squeezing parameter. Phys. Rev. Lett., 122:090503, Mar 2019. DOI: https://doi.org/10.1103/PhysRevLett.122.090503.

[32] D. J. Wineland, J. J. Bollinger, W. M. Itano, F. L. Moore, and D. J. Heinzen. Spin squeezing and reduced quantum noise in spectroscopy. Phys. Rev. A, 46:R6797-R6800, Dec 1992. DOI: https://doi.org/10.1103/PhysRevA.46.R6797.

[33] D. J. Wineland, J. J. Bollinger, W. M. Itano, and D. J. Heinzen. Squeezed atomic states and projection noise in spectroscopy. Phys. Rev. A, 50:67-88, Jul 1994. DOI: https://doi.org/10.1103/PhysRevA.50.67.

[34] D. Leibfried, E. Knill, S. Seidelin, J. Britton, R. B. Blakestad, J. Chiaverini, D. B. Hume, W. M. Itano, J. D. Jost, C. Langer, R. Ozeri, R. Reichle, and D. J. Wineland. Creation of a six-atom 'Schrödinger cat' state. Nature, 438(7068):639-642, Dec 2005. DOI: https://doi.org/10.1038/nature04251.

[35] H. Strobel, W. Muessel, D. Linnemann, T. Zibold, D. B. Hume, L. Pezze, A. Smerzi, and M. K. Oberthaler. Fisher information and entanglement of non-Gaussian spin states. Science, 345(6195):424-427, Jul $2014 . \quad$ DOI: https://doi.org/10.1126/science.1250147.

[36] L. Pezzé and A. Smerzi. Entanglement, nonlinear dynamics, and the Heisenberg limit. Phys. Rev. Lett., 102:100401, Mar 2009. DOI: https://doi.org/10.1103/PhysRevLett.102.100401.

[37] D. Kielpinski, V. Meyer, M. A. Rowe, C. A. Sackett, W. M. Itano, C. Monroe, and D. J. Wineland. A decoherence-free quantum memory using trapped ions. Science, 291(5506):1013-1015, Jan 2001. DOI: https://doi.org/10.1126/science.1057357.

C. F. Roos, M. Chwalla, K. Kim, M. Riebe, and R. Blatt. 'Designer atoms' for quantum metrology. $\mathrm{Na}$ ture, 443(7109):316-319, Sep 2006. DOI: https://doi.org/10.1038/nature05101.

9] G. S. Agarwal, R. R. Puri, and R. P. Singh. Atomic Schrödinger cat states. Phys. Rev. A, 56:2249-2254, Sep 1997. DOI: https://doi.org/10.1103/PhysRevA.56.2249.

[40] J. P. Dowling, G. S. Agarwal, and W. P. Schleich. Wigner distribution of a general angular-momentum state: Applications to a collection of two-level atoms. Phys. Rev. A, 49:4101-4109, May 1994. DOI: https://doi.org/10.1103/PhysRevA.49.4101.

[41] D. Gottesman, A. Kitaev, and J. Preskill. Encoding a qubit in an oscillator. Phys. Rev. A, 64:012310, Jun 2001. DOI: https://doi.org/10.1103/PhysRevA.64.012310.

[42] K. Duivenvoorden, B. M. Terhal, and D. Weigand. Single-mode displacement sensor. Phys. Rev. A, 95:012305, Jan 2017. DOI: https://doi.org/10.1103/PhysRevA.95.012305.

[43] G. S. Agarwal. Relation between atomic coherent-state representation, state multipoles, and generalized phase-space distributions. Phys. Rev. A, 24:2889-2896, Dec 1981. DOI: https://doi.org/10.1103/PhysRevA.24.2889.

[44] M. J. W. Hall and H. M. Wiseman. Does nonlinear metrology offer improved resolution? Answers from quantum information theory. Phys. Rev. X, 2:041006, Oct 2012. DOI: https://doi.org/10.1103/PhysRevX.2.041006.

[45] M. Schulte, C. Lisdat, P. O. Schmidt, U. Sterr, and K. Hammerer. Prospects and challenges for squeezing-enhanced optical atomic clocks. arXiv e-prints, art. arXiv:1911.00882, Nov 2019.

[46] F. T. Arecchi, E. Courtens, R. Gilmore, and H. Thomas. Atomic coherent states in quantum optics. Phys. Rev. A, 6:2211-2237, Dec 1972. DOI: https://doi.org/10.1103/PhysRevA.6.2211.

[47] C. W. Helstrom. Quantum detection and estimation theory. Journal of Statisti- 
cal Physics, 1(2):231-252, $1969 . \quad$ DOI: https://doi.org/10.1007/bf01007479.

[48] S. L. Braunstein and C. M. Caves.
Statistical distance and the geometry of quantum states. Phys. Rev. Lett., 72:3439-3443, May 1994. DOI: https://doi.org/10.1103/PhysRevLett.72.3439. 


\section{A Signal and noise from spin characteristic functions}

The matrices $M$ and $Q$ can be conveniently evaluated from collective spin expectation values when transforming to a spherical basis $S_{+}, S_{z}, S_{-}$and using the characteristic function approach of [46]. For the signal strength, we find

$$
M=\left(\begin{array}{ccc}
\frac{1}{2}\left[n_{1}+n_{2}\right] & 0 & 0 \\
0 & \frac{1}{2}\left[n_{1}-n_{2}\right] & n_{3} \\
0 & n_{4} & 0
\end{array}\right)
$$

with

$$
\begin{aligned}
& n_{1}=\frac{N(N-1)}{2} \sin \left(\frac{\mu-\nu}{2}\right) \cos ^{N-2}\left(\frac{\mu-\nu}{2}\right) \\
& n_{2}=-\frac{N(N-1)}{2} \sin \left(\frac{\mu-\nu}{2}\right) \cos ^{N-2}\left(\frac{\mu+\nu}{2}\right) \\
& n_{3}=-\frac{N}{2} \cos ^{N-1}\left(\frac{\mu}{2}\right) \\
& n_{4}=\frac{N}{2} \cos ^{N-1}\left(\frac{\nu}{2}\right) .
\end{aligned}
$$

For the spin covariances, the respective matrix is

$$
Q=\left(\begin{array}{ccc}
\frac{1}{2}\left[q_{1}+q_{2}\right]-q_{0}^{2} & 0 & 0 \\
0 & \frac{1}{2}\left[q_{1}-q_{2}\right] & q_{3} \\
0 & q_{3} & q_{4}
\end{array}\right)
$$

with

$$
\begin{aligned}
& q_{0}=\frac{N}{2} \cos ^{N-1}\left(\frac{\nu}{2}\right), \quad q_{1}=\frac{N(N+1)}{4} \\
& q_{2}=\frac{N(N-1)}{4} \cos ^{N-2}(\nu) \\
& q_{3}=\frac{N(N-1)}{4} \sin \left(\frac{\nu}{2}\right) \cos ^{N-2}\left(\frac{\nu}{2}\right) \\
& q_{4}=\frac{N}{4} .
\end{aligned}
$$

We show details of the derivation in the rest of this section and extend the calculations to collective and individual dephasing in the next section.

The spherical basis $S_{+}, S_{z}, S_{-}$for a collective spin of length $S=N / 2$ includes the angular momentum ladder operators $S_{ \pm}=S_{x} \pm i S_{y}$. Correspondingly, $S_{x}=\frac{1}{2}\left(S_{+}+S_{-}\right), S_{y}=\frac{1}{2 i}\left(S_{+}-S_{-}\right)$and

$$
\left[S_{+}, S_{-}\right]=2 S_{z}, \quad\left[S_{z}, S_{ \pm}\right]= \pm S_{ \pm}
$$

In the following we aim at calculating expectation values with respect to spin coherent states $|\theta, \varphi\rangle=e^{-i \theta\left(S_{x} \sin \varphi-S_{y} \cos \varphi\right)}|-S\rangle_{z}$, where the special case $\varphi=0, \theta=\pi / 2$ is the initial state $|\mathrm{x}\rangle$ of a standard Ramsey protocol after the first $\pi / 2$-pulse. Anti-normally ordered expectation values in the spherical basis, meaning that $S_{-}$is always left of $S_{z}$ and both to the left of $S_{+}$, of the general form $\left\langle\theta, \varphi\left|S_{-}^{c} e^{\mathfrak{c} S_{-}} S_{z}^{b} e^{\mathfrak{b} S_{z}} S_{+}^{a} e^{\mathfrak{a} S_{+}}\right| \theta, \varphi\right\rangle$, with integers $a, b, c$ as well as arbitrary coefficients $\mathfrak{a}, \mathfrak{b}, \mathfrak{c}$, can be calculated via derivatives of a spin characteristic function [46]. Explicitly,

$$
\left\langle\theta, \varphi\left|S_{-}^{c} e^{\mathfrak{c} S_{-}} S_{z}^{b} e^{\mathfrak{b} S_{z}} S_{+}^{a} e^{\mathfrak{a} S_{+}}\right| \theta, \varphi\right\rangle=\left.\partial_{\alpha}^{a} \partial_{\beta}^{b} \partial_{\gamma}^{c} X_{A}(\alpha, \beta, \gamma)\right|_{\alpha=\mathfrak{a}, \beta=\mathfrak{b}, \gamma=\mathfrak{c}}
$$

with the anti-normally ordered spin characteristic function [46]

$$
\begin{aligned}
X_{A}(\alpha, \beta, \gamma) & =\left\langle\theta, \varphi\left|e^{\gamma S_{-}} e^{\beta S_{z}} e^{\alpha S_{+}}\right| \theta, \varphi\right\rangle \\
& =\left[e^{-\beta / 2} \cos ^{2}(\theta / 2)+e^{\beta / 2}\left(\sin (\theta / 2) e^{-i \varphi}+\alpha \cos (\theta / 2)\right)\left(\sin (\theta / 2) e^{i \varphi}+\gamma \cos (\theta / 2)\right)\right]^{2 S} .
\end{aligned}
$$


Using the transformation matrix

$$
A=\left(\begin{array}{ccc}
\frac{1}{2} & 0 & \frac{1}{2} \\
\frac{1}{2 i} & 0 & -\frac{1}{2 i} \\
0 & 1 & 0
\end{array}\right) \quad \text { and } \quad M_{s}=i\left(\begin{array}{ccc}
\left\langle\left[S_{+}(\mu), S_{+}(\nu)\right]\right\rangle & \left\langle\left[S_{+}(\mu), S_{z}(\nu)\right]\right\rangle & \left\langle\left[S_{+}(\mu), S_{-}(\nu)\right]\right\rangle \\
\left\langle\left[S_{z}(\mu), S_{+}(\nu)\right]\right\rangle & \left\langle\left[S_{z}(\mu), S_{z}(\nu)\right]\right\rangle & \left\langle\left[S_{z}(\mu), S_{-}(\nu)\right]\right\rangle \\
\left\langle\left[S_{-}(\mu), S_{+}(\nu)\right]\right\rangle & \left\langle\left[S_{-}(\mu), S_{z}(\nu)\right]\right\rangle & \left\langle\left[S_{-}(\mu), S_{-}(\nu)\right]\right\rangle
\end{array}\right),
$$

the matrix $M$ is related to its counterpart $M_{s}$ in the spherical basis via $M=A M_{s} A^{T}$. In the same way the covariance variance $Q$ can be expressed as $Q=A Q_{s} A^{T}$ with

$$
Q_{s}=\frac{1}{2}\left(\begin{array}{lll}
\left\langle\left[S_{+}(\nu), S_{+}(\nu)\right]_{+}\right\rangle & \left\langle\left[S_{+}(\nu), S_{z}(\nu)\right]_{+}\right\rangle & \left\langle\left[S_{+}(\nu), S_{-}(\nu)\right]_{+}\right\rangle \\
\left\langle\left[S_{z}(\nu), S_{+}(\nu)\right]_{+}\right\rangle & \left\langle\left[S_{z}(\nu), S_{z}(\nu)\right]_{+}\right\rangle & \left\langle\left[S_{z}(\nu), S_{-}(\nu)\right]_{+}\right\rangle \\
\left\langle\left[S_{-}(\nu), S_{+}(\nu)\right]_{+}\right\rangle & \left\langle\left[S_{-}(\nu), S_{z}(\nu)\right]_{+}\right\rangle & \left\langle\left[S_{-}(\nu), S_{-}(\nu)\right]_{+}\right\rangle
\end{array}\right)-\vec{j} \vec{j}^{T}
$$

where $\vec{j}=\left(\left\langle S_{+}(\nu)\right\rangle,\left\langle S_{z}(\nu)\right\rangle,\left\langle S_{-}(\nu)\right\rangle\right)^{T}$ and $[\cdot, \cdot]_{+}$denotes the anti-commutator.

To calculate the expectation values therein, they have to be brought into anti-normal order before using Eq. (13). For this we use that the transformed operators $S_{(+, z,-)}(\mu)=T_{\mu}^{\dagger} S_{(+, z,-)} T_{\mu}$ are $S_{z}(\mu)=$ $S_{z}$ and

$$
S_{ \pm}(\mu)=e^{i \mu / 2} S_{ \pm} e^{ \pm i \mu S_{z}}=e^{-i \mu / 2} e^{ \pm i \mu S_{z}} S_{ \pm} .
$$

Furthermore, the transformations

$$
\begin{aligned}
& e^{i \mu S_{z}} S_{ \pm} e^{-i \mu S_{z}}=e^{ \pm i \mu} S_{ \pm}, \quad e^{-i \mu S_{z}} S_{ \pm} e^{i \mu S_{z}}=e^{\mp i \mu} S_{ \pm} \\
& \Rightarrow S_{+} e^{ \pm i \mu S_{z}}=e^{\mp i \mu} e^{ \pm i \mu S_{z}} S_{+}, \quad e^{ \pm i \mu S_{z}} S_{-}=e^{\mp i \mu} S_{-} e^{ \pm i \mu S_{z}}
\end{aligned}
$$

are applied to obtain anti-normal ordering. With this, we find for the first order moments

$$
\begin{aligned}
\left\langle S_{+}(\nu)\right\rangle & =e^{-i \nu / 2}\left\langle e^{i \nu S_{z}} S_{+}\right\rangle=\left.e^{-i \nu / 2} \partial_{\alpha} X_{A}\right|_{\alpha=\gamma=0, \beta=i \nu} \\
\left\langle S_{z}(\nu)\right\rangle & =\left\langle S_{z}\right\rangle=\left.\partial_{\beta} X_{A}\right|_{\alpha=\beta=\gamma=0} \\
\left\langle S_{-}(\nu)\right\rangle & =e^{i \nu / 2}\left\langle S_{-} e^{-i \nu S_{z}}\right\rangle=\left.e^{i \nu / 2} \partial_{\gamma} X_{A}\right|_{\alpha=\gamma=0, \beta=-i \nu}
\end{aligned}
$$

and all expectation values are with respect to the coherent spin state $|\theta, \varphi\rangle$. For the symmetric second order moments:

$$
\begin{aligned}
\left\langle\left[S_{+}(\nu), S_{+}(\nu)\right]_{+}\right\rangle & =2\left\langle S_{+}(\nu) S_{+}(\nu)\right\rangle \\
& =2 e^{-i 2 \nu}\left\langle e^{i 2 \nu S_{z}} S_{+}^{2}\right\rangle \\
& =\left.2 e^{-i 2 \nu} \partial_{\alpha} \partial_{\alpha} X_{A}\right|_{\alpha=\gamma=0, \beta=i 2 \nu} \\
\left\langle\left[S_{+}(\nu), S_{z}(\nu)\right]_{+}\right\rangle & =\left\langle S_{+}(\nu) S_{z}+S_{z} S_{+}(\nu)\right\rangle \\
& =e^{-i \nu / 2}\left\langle 2 S_{z} e^{i \nu S_{z}} S_{+}-e^{i \nu S_{z}} S_{+}\right\rangle \\
& =e^{-i \nu / 2}\left\{\left.2 \partial_{\beta} \partial_{\alpha} X_{A}\right|_{\alpha=\gamma=0, \beta=i \nu}-\left.\partial_{\alpha} X_{A}\right|_{\alpha=\gamma=0, \beta=i \nu}\right\} \\
\left\langle\left[S_{+}(\nu), S_{-}(\nu)\right]_{+}\right\rangle & =\left\langle\left[S_{+}, S_{-}\right]_{+}(\nu)\right\rangle \\
& =2\left\langle S_{-} S_{+}+S_{z}\right\rangle \\
& =2\left\{\left.\partial_{\gamma} \partial_{\alpha} X_{A}\right|_{\alpha=\beta=\gamma=0}+\left.\partial_{\beta} X_{A}\right|_{\alpha=\beta=\gamma=0}\right\} \\
\left\langle\left[S_{z}(\nu), S_{+}(\nu)\right]_{+}\right\rangle & =\left\langle\left[S_{+}(\nu), S_{z}(\nu)\right]_{+}\right\rangle \\
\left\langle\left[S_{z}(\nu), S_{z}(\nu)\right]_{+}\right\rangle & =2\left\langle S_{z}^{2}\right\rangle \\
& =\left.2 \partial_{\beta} \partial_{\beta} X_{A}\right|_{\alpha=\beta=\gamma=0} \\
\left\langle\left[S_{z}(\nu), S_{-}(\nu)\right]_{+}\right\rangle & =\left\langle S_{z} S_{-}(\nu)+S_{-}(\nu) S_{z}\right\rangle \\
& =e^{i \nu / 2}\left\langle 2 S_{-} S_{z} e^{-i \nu S_{z}}-S_{-} e^{-i \nu S_{z}}\right\rangle \\
& =e^{i \nu / 2}\left\{\left.2 \partial_{\beta} \partial_{\gamma} X_{A}\right|_{\alpha=\gamma=0, \beta=-i \nu}-\left.\partial_{\gamma} X_{A}\right|_{\alpha=\gamma=0, \beta=-i \nu}\right\}
\end{aligned}
$$




$$
\begin{aligned}
\left\langle\left[S_{-}(\nu), S_{+}(\nu)\right]_{+}\right\rangle & =\left\langle\left[S_{+}(\nu), S_{-}(\nu)\right]_{+}\right\rangle \\
\left\langle\left[S_{-}(\nu), S_{z}(\nu)\right]_{+}\right\rangle & =\left\langle\left[S_{z}(\nu), S_{-}(\nu)\right]_{+}\right\rangle \\
\left\langle\left[S_{-}(\nu), S_{-}(\nu)\right]_{+}\right\rangle & =2\left\langle S_{-}(\nu) S_{-}(\nu)\right\rangle \\
& =2 e^{i 2 \nu}\left\langle S_{-}^{2} e^{-i 2 \nu S_{z}}\right\rangle \\
& =\left.2 e^{i 2 \nu} \partial_{\gamma} \partial_{\gamma} X_{A}\right|_{\alpha=\gamma=0, \beta=-i 2 \nu}
\end{aligned}
$$

Finally, the moments for the commutators are:

$$
\begin{aligned}
\left\langle\left[S_{+}(\mu), S_{+}(\nu)\right]\right\rangle & =\left\langle S_{+}(\mu) S_{+}(\nu)-S_{+}(\nu) S_{+}(\mu)\right\rangle \\
& =e^{-i(\mu+\nu) / 2}\left(e^{-i \nu}-e^{-i \mu}\right)\left\langle e^{i(\mu+\nu) S_{z}} S_{+}^{2}\right\rangle \\
& =\left.e^{-i(\mu+\nu) / 2}\left(e^{-i \nu}-e^{-i \mu}\right) \partial_{\alpha} \partial_{\alpha} X_{A}\right|_{\alpha=\gamma=0, \beta=i(\mu+\nu)} \\
\left\langle\left[S_{+}(\mu), S_{z}(\nu)\right]\right\rangle & =\left\langle S_{+}(\mu) S_{z}-S_{z} S_{+}(\mu)\right\rangle \\
& =-e^{-i \mu / 2}\left\langle e^{i \mu S_{z}} S_{+}\right\rangle \\
& =-\left.e^{-i \mu / 2} \partial_{\alpha} X_{A}\right|_{\alpha=\gamma=0, \beta=i \mu}
\end{aligned}
$$

$$
\begin{aligned}
\left\langle\left[S_{+}(\mu), S_{-}(\nu)\right]\right\rangle & =\left\langle S_{+}(\mu) S_{-}(\nu)-S_{-}(\nu) S_{+}(\mu)\right\rangle \\
& =e^{-i(\mu-\nu) / 2}\left\langle 2 S_{z} e^{i(\mu-\nu) S_{z}}+\left(e^{-i(\mu-\nu)}-1\right) S_{-} e^{i(\mu-\nu) S_{z}} S_{+}\right\rangle \\
& =e^{-i(\mu-\nu) / 2}\left\{\left.2 \partial_{\beta} X_{A}\right|_{\alpha=\gamma=0, \beta=i(\mu-\nu)}+\left.\left(e^{-i(\mu-\nu)}-1\right) \partial_{\gamma} \partial_{\alpha} X_{A}\right|_{\alpha=\gamma=0, \beta=i(\mu-\nu)}\right\} \\
\left\langle\left[S_{z}(\mu), S_{+}(\nu)\right]\right\rangle & =\left\langle S_{z} S_{+}(\nu)-S_{+}(\nu) S_{z}\right\rangle \\
& =-\left\langle\left[S_{+}(\nu), S_{z}\right]\right\rangle \\
& =\left.e^{-i \nu / 2} \partial_{\alpha} X_{A}\right|_{\alpha=\gamma=0, \beta=i \nu}
\end{aligned}
$$

$$
\begin{aligned}
\left\langle\left[S_{z}(\mu), S_{-}(\nu)\right]\right\rangle & =\left\langle S_{z} S_{-}(\nu)-S_{-}(\nu) S_{z}\right\rangle \\
& =-e^{i \nu / 2}\left\langle S_{-} e^{-i \nu S_{z}}\right\rangle \\
& =-\left.e^{i \nu / 2} \partial_{\gamma} X_{A}\right|_{\alpha=\gamma=0, \beta=-i \nu}
\end{aligned}
$$

$$
\begin{gathered}
\left\langle\left[S_{-}(\mu), S_{+}(\nu)\right]\right\rangle=\left\langle S_{-}(\mu) S_{+}(\nu)-S_{+}(\nu) S_{-}(\mu)\right\rangle \\
=e^{i(\mu-\nu) / 2}\left\langle\left(1-e^{i(\mu-\nu)}\right) S_{-} e^{-i(\mu-\nu) S_{z}} S_{+}-2 S_{z} e^{-i(\mu-\nu) S_{z}}\right\rangle \\
=e^{i(\mu-\nu) / 2}\left\{\left.\left(1-e^{i(\mu-\nu)}\right) \partial_{\alpha} \partial_{\gamma} X_{A}\right|_{\alpha=\gamma=0, \beta=-i(\mu-\nu)}-\left.2 \partial_{\beta} X_{A}\right|_{\alpha=\gamma=0, \beta=-i(\mu-\nu)}\right\} \\
\left\langle\left[S_{-}(\mu), S_{z}(\nu)\right]\right\rangle \\
=-\left\langle\left[S_{z}, S_{-}(\mu)\right]\right\rangle \\
=e^{i \mu / 2}\left\langle S_{-} e^{-i \mu S_{z}}\right\rangle \\
=\left.e^{i \mu / 2} \partial_{\gamma} X_{A}\right|_{\alpha=\gamma=0, \beta=-i \mu}
\end{gathered}
$$

$$
\begin{aligned}
\left\langle\left[S_{-}(\mu), S_{-}(\nu)\right]\right\rangle & =\left\langle S_{-}(\mu) S_{-}(\nu)-S_{-}(\nu) S_{-}(\mu)\right\rangle \\
& =e^{i(\mu+\nu) / 2}\left(e^{i \mu}-e^{i \nu}\right)\left\langle S_{-}^{2} e^{-i(\mu+\nu) S_{z}}\right\rangle \\
& =\left.e^{i(\mu+\nu) / 2}\left(e^{i \mu}-e^{i \nu}\right) \partial_{\gamma} \partial_{\gamma} X_{A}\right|_{\alpha=\gamma=0, \beta=-i(\mu+\nu)}
\end{aligned}
$$


With the characteristic function

$$
X_{A}(0, \pi / 2)=\left[\frac{1}{2} e^{-\beta / 2}+\frac{1}{2} e^{\beta / 2}(1+\alpha)(1+\gamma)\right]^{2 S}
$$

we then find

$$
\left(\begin{array}{c}
\left\langle S_{+}(\nu)\right\rangle \\
\left\langle S_{z}(\nu)\right\rangle \\
\left\langle S_{-}(\nu)\right\rangle
\end{array}\right)=\left(\begin{array}{c}
q_{0} \\
0 \\
q_{0}
\end{array}\right)
$$

with $q_{0}=S \cos ^{2 S-1}\left(\frac{\nu}{2}\right)$. Likewise one finds

$$
Q_{s}=\left(\begin{array}{ccc}
q_{2} & i q_{3} & q_{1} \\
i q_{3} & q_{4} & -i q_{3} \\
q_{1} & -i q_{3} & q_{2}
\end{array}\right)-\left(\begin{array}{c}
q_{0} \\
0 \\
q_{0}
\end{array}\right)\left(q_{0}, 0, q_{0}\right)
$$

and thus

$$
Q=A Q_{s} A^{T}=\left(\begin{array}{ccc}
\frac{1}{2}\left[q_{1}+q_{2}\right]-q_{0}^{2} & 0 & 0 \\
0 & \frac{1}{2}\left[q_{1}-q_{2}\right] & q_{3} \\
0 & q_{3} & q_{4}
\end{array}\right)
$$

as well as

$$
M_{s}=\left(\begin{array}{ccc}
n_{2} & i n_{3} & n_{1} \\
i n_{4} & 0 & -i n_{4} \\
n_{1} & -i n_{3} & n_{2}
\end{array}\right) \quad \text { and } \quad M=A M_{s} A^{T}=\left(\begin{array}{ccc}
\frac{1}{2}\left[n_{1}+n_{2}\right] & 0 & 0 \\
0 & \frac{1}{2}\left[n_{1}-n_{2}\right] & n_{3} \\
0 & n_{4} & 0
\end{array}\right)
$$

in accord to the results stated initially.

\section{B Expectation values with dephasing}

This section contains details on calculating spin expectation values with dephasing noise. The OAT dynamics in the case of collective dephasing is given by the master equation

$$
\dot{\rho}=-i[H, \rho]+\gamma_{C} \mathcal{L}_{C}[\rho]
$$

with $H=\chi S_{z}^{2}, \mathcal{L}_{C}[\rho]=S_{z} \rho S_{z}-\frac{1}{2} S_{z}^{2} \rho-\frac{1}{2} \rho S_{z}^{2}$ and the dephasing rate $\gamma_{C}$. Likewise, individual dephasing is described by the master equation

$$
\dot{\rho}=-i[H, \rho]+\gamma_{I} \mathcal{L}_{I}[\rho]
$$

with $H=\chi S_{z}^{2}$ and $\mathcal{L}_{I}[\rho]=\sum_{k=1}^{N} \sigma_{z}^{k} \rho \sigma_{z}^{k}-\rho$ where $\gamma_{I}>0$ is the individual dephasing rate, equal for all particles. The formal solution for collective dephasing is

$$
\rho(t)=T_{\mu} e^{\sigma \frac{|\mu|}{2} \mathcal{L}_{C}}\left[\rho_{0}\right] T_{\mu}^{\dagger}=e^{\sigma \frac{|\mu|}{2} \mathcal{L}_{C}}\left[T_{\mu} \rho_{0} T_{\mu}^{\dagger}\right]
$$

from an initial state $\rho_{0}$ and with $\sigma=\left|\gamma_{C}\right| /|\chi|$. For individual dephasing we find

$$
\rho(t)=T_{\mu} e^{\Sigma \frac{|\mu|}{2} \mathcal{L}_{I}}\left[\rho_{0}\right] T_{\mu}^{\dagger}=e^{\Sigma \frac{|\mu|}{2} \mathcal{L}_{I}}\left[T_{\mu} \rho_{0} T_{\mu}^{\dagger}\right]
$$

where $\Sigma=\left|\gamma_{I}\right| /|\chi|$. Expectation values of any operator $A$ are then

$$
\langle A\rangle=\operatorname{tr}\{A \rho(t)\}=\operatorname{tr}\left\{A e^{\sigma \frac{|\mu|}{2} \mathcal{L}_{k}}\left[T_{\mu} \rho_{0} T_{\mu}^{\dagger}\right]\right\}=\operatorname{tr}\left\{T_{\mu}^{\dagger} e^{\left.\sigma \frac{|\mu|}{2} \mathcal{L}_{k}^{\dagger}[A] T_{\mu} \rho_{0}\right\} .}\right.
$$

Here, $\mathcal{L}^{\dagger}$ is the adjoint Lindblad operator, defined via

$$
\operatorname{tr}\{A \mathcal{L}[B]\}=\operatorname{tr}\left\{\mathcal{L}^{\dagger}[A] B\right\}
$$


so that

$$
\mathcal{L}^{\dagger}[A]=L^{\dagger} A L-\frac{1}{2} L^{\dagger} L A-\frac{1}{2} A L^{\dagger} L
$$

given

$$
\mathcal{L}[A]=L A L^{\dagger}-\frac{1}{2} L^{\dagger} L A-\frac{1}{2} A L^{\dagger} L .
$$

For both, collective and individual dephasing, this simplifies to $\mathcal{L}_{C}^{\dagger}=\mathcal{L}_{C}$ and $\mathcal{L}_{I}^{\dagger}=\mathcal{L}_{I}$. For the protocols of the main text this allows to evaluate the expectation values

$$
\left.\left\langle S_{-}^{k_{-}} S_{z}^{k_{z}} S_{+}^{k_{+}}\right\rangle\right|_{\phi=0}=\operatorname{tr}\left\{T_{\nu}^{\dagger} e^{\sigma \frac{|\mu|}{2} \mathcal{L}}\left[e^{\sigma \frac{|\nu-\mu|}{2} \mathcal{L}}\left[S_{-}^{k_{-}} S_{z}^{k_{z}} S_{+}^{k_{+}}\right]\right] T_{\nu} \rho_{0}\right\}
$$

required for the spin covariance matrix as well as the slope

$$
\begin{aligned}
\left.\frac{\partial\left\langle S_{\vec{m}}\right\rangle}{\partial \phi}\right|_{\phi=0} & =\frac{\partial}{\partial \phi}\left(\sum_{k} \operatorname{tr}\left\{m_{k} S_{k} e^{\sigma \frac{|\nu-\mu|}{2} \mathcal{L}}\left[T_{\nu-\mu} e^{-i \phi \sum_{l} n_{l} S_{l}} e^{\sigma \frac{|\mu|}{2} \mathcal{L}}\left[T_{\mu} \rho_{0} T_{\mu}^{\dagger}\right] e^{i \phi \sum_{l} n_{l} S_{l}} T_{\nu-\mu}^{\dagger}\right]\right\}\right)_{\left.\right|_{\phi=0}} \\
& =i \sum_{l, k} n_{l} M_{l k} m_{k}
\end{aligned}
$$

with

$$
M_{l, k}=\operatorname{tr}\left\{T_{\mu}^{\dagger} e^{\sigma \frac{|\mu|}{2} \mathcal{L}}\left[\left[S_{l}, T_{\nu-\mu}^{\dagger} e^{\sigma \frac{|\nu-\mu|}{2}} \mathcal{L}\left[S_{k}\right] T_{\nu-\mu}\right]\right] T_{\mu} \rho\right\} .
$$

(The same applies for individual dephasing with $\sigma \leftrightarrow \Sigma$ and $\mathcal{L} \leftrightarrow \mathcal{L}^{\prime}$.) The expectation values presented here can now be reduced to their noiseless version by explicitly evaluating the transformed operators. At this point however we have to separate collective and individual dephasing. For collective dephasing the following holds: First, it is clear that $e^{\sigma \frac{|\mu|}{2}} \mathcal{L}\left[S_{z}\right]=S_{z}$ and from the commutation relations (12) it follows that $e^{\sigma \frac{|\mu|}{2}} \mathcal{L}\left[S_{ \pm}\right]=e^{-\sigma \frac{|\mu|}{4}} S_{ \pm}$. Repeated application of the commutation relations then also gives

$$
\begin{aligned}
& e^{\sigma \frac{|\mu|}{2} \mathcal{L}}\left[S_{ \pm}^{2}\right]=e^{-\sigma|\mu|} S_{ \pm}^{2} \\
& \left.e^{\sigma \frac{|\mu|}{2}} \mathcal{L}_{\left[S_{ \pm}\right.} S_{z}\right]=e^{-\sigma \frac{|\mu|}{4}} S_{ \pm} S_{z} \\
& e^{\sigma \frac{|\mu|}{2} \mathcal{L}}\left[S_{z} S_{ \pm}\right]=e^{-\sigma \frac{|\mu|}{4}} S_{z} S_{ \pm} \\
& e^{\sigma \frac{|\mu|}{2} \mathcal{L}}\left[S_{ \pm} S_{\mp}\right]=S_{ \pm} S_{\mp}
\end{aligned}
$$

which allows to express all expectation values to the ones with $\sigma=0$ and appropriate exponential damping factors. With this, we find

$$
\left(\begin{array}{c}
\left\langle S_{+}(\nu)\right\rangle \\
\left\langle S_{z}(\nu)\right\rangle \\
\left\langle S_{-}(\nu)\right\rangle
\end{array}\right)=\left(\begin{array}{c}
\tilde{q}_{0} \\
0 \\
\tilde{q}_{0}
\end{array}\right)
$$

with, again, $\tilde{q}_{0}=e^{-\sigma \frac{|\nu-\mu|+|\mu|}{4}} S \cos ^{2 S-1}\left(\frac{\nu}{2}\right)$.

Likewise one finds

$$
\tilde{Q}_{s}=\left(\begin{array}{ccc}
\tilde{q}_{2} & i \tilde{q}_{3} & \tilde{q}_{1} \\
i \tilde{q}_{3} & \tilde{q}_{4} & -i \tilde{q}_{3} \\
\tilde{q}_{1} & -i \tilde{q}_{3} & \tilde{q}_{2}
\end{array}\right)-\left(\begin{array}{c}
\tilde{q}_{0} \\
0 \\
\tilde{q}_{0}
\end{array}\right)\left(\tilde{q}_{0}, 0, \tilde{q}_{0}\right)
$$

and thus

$$
\tilde{Q}=A \tilde{Q}_{s} A^{T}=\left(\begin{array}{ccc}
\frac{1}{2}\left[\tilde{q}_{1}+\tilde{q}_{2}\right]-\tilde{q}_{0}^{2} & 0 & 0 \\
0 & \frac{1}{2}\left[\tilde{q}_{1}-\tilde{q}_{2}\right] & \tilde{q}_{3} \\
0 & \tilde{q}_{3} & \tilde{q}_{4}
\end{array}\right)
$$


with

$$
\begin{aligned}
& \tilde{q}_{1}=q_{1}=S^{2}+\frac{S}{2}, \quad \tilde{q}_{2}=e^{-\sigma(|\nu-\mu|+|\mu|)} \frac{S}{2}(2 S-1) \cos (\nu)^{2 S-2}, \\
& \tilde{q}_{3}=e^{-\sigma \frac{|\nu-\mu|+|\mu|}{4}} \frac{S}{2}(2 S-1) \cos \left(\frac{\nu}{2}\right)^{2 S-2} \sin \left(\frac{\nu}{2}\right) \quad \text { and } \quad \tilde{q}_{4}=q_{4}=\frac{S}{2} .
\end{aligned}
$$

Finally,

$$
\tilde{M}_{s}=\left(\begin{array}{ccc}
\tilde{n}_{2} & i \tilde{n}_{3} & \tilde{n}_{1} \\
i \tilde{n}_{4} & 0 & -i \tilde{n}_{4} \\
\tilde{n}_{1} & -i \tilde{n}_{3} & \tilde{n}_{2}
\end{array}\right) \quad \text { and } \quad \tilde{M}=A \tilde{M}_{s} A^{T}=\left(\begin{array}{ccc}
\frac{1}{2}\left[\tilde{n}_{1}+\tilde{n}_{2}\right] & 0 & 0 \\
0 & \frac{1}{2}\left[\tilde{n}_{1}-\tilde{n}_{2}\right] & \tilde{n}_{3} \\
0 & \tilde{n}_{4} & 0
\end{array}\right)
$$

with

$\tilde{n}_{1}=e^{-\sigma \frac{|\nu-\mu|}{4}} S(2 S-1) \sin \left(\frac{\mu-\nu}{2}\right) \cos \left(\frac{\mu-\nu}{2}\right)^{2 S-2}, \quad \tilde{n}_{2}=-e^{-\sigma\left(\frac{|\nu-\mu|}{4}+|\mu|\right)} S(2 S-1) \sin \left(\frac{\mu-\nu}{2}\right) \cos \left(\frac{\mu+\nu}{2}\right)^{2 S-2}$,

$$
\tilde{n}_{3}=-e^{-\sigma \frac{|\mu|}{4}} S \cos \left(\frac{\mu}{2}\right)^{2 S-1} \quad \text { and } \quad \tilde{n}_{4}=e^{-\sigma \frac{|\nu-\mu|+|\mu|}{4}} S \cos \left(\frac{\nu}{2}\right)^{2 S-1} .
$$

A similar study shows that for individual dephasing the operators transform as

$$
\begin{aligned}
& e^{\Sigma \frac{|\mu|}{2} \mathcal{L}^{\prime}}\left[S_{z}\right]=S_{z} \\
& e^{\Sigma \frac{|\mu|}{2} \mathcal{L}^{\prime}}\left[S_{ \pm}\right]=e^{-\Sigma|\mu|} S_{ \pm} \\
& e^{\Sigma \frac{|\mu|}{2} \mathcal{L}^{\prime}}\left[S_{ \pm}^{2}\right]=e^{-2 \Sigma|\mu|} S_{ \pm}^{2} \\
& e^{\Sigma \frac{|\mu|}{2} \mathcal{L}^{\prime}}\left[S_{ \pm} S_{z}\right]=e^{-\Sigma|\mu|} S_{ \pm} S_{z} \\
& e^{\Sigma \frac{|\mu|}{2} \mathcal{L}^{\prime}}\left[S_{z} S_{ \pm}\right]=e^{-\Sigma|\mu|} S_{z} S_{ \pm} \\
& e^{\Sigma \frac{|\mu|}{2} \mathcal{L}^{\prime}}\left[S_{ \pm} S_{\mp}\right]=e^{-2 \Sigma|\mu|}\left[S_{ \pm} S_{\mp}+\left(\frac{N}{2} \pm S_{z}\right)\left(e^{2 \Sigma|\mu|}-1\right)\right]
\end{aligned}
$$

With this, we find

$$
\left(\begin{array}{c}
\left\langle S_{+}(\nu)\right\rangle \\
\left\langle S_{z}(\nu)\right\rangle \\
\left\langle S_{-}(\nu)\right\rangle
\end{array}\right)=\left(\begin{array}{c}
q_{0}^{\prime} \\
0 \\
q_{0}^{\prime}
\end{array}\right)
$$

with $q_{0}^{\prime}=e^{-\Sigma(|\nu-\mu|+|\mu|)} q_{0}$.

Further

and thus

$$
Q_{s}^{\prime}=\left(\begin{array}{ccc}
q_{2}^{\prime} & i q_{3}^{\prime} & q_{1}^{\prime} \\
i q_{3}^{\prime} & q_{4}^{\prime} & -i q_{3}^{\prime} \\
q_{1}^{\prime} & -i q_{3}^{\prime} & q_{2}^{\prime}
\end{array}\right)-\left(\begin{array}{c}
q_{0}^{\prime} \\
0 \\
q_{0}^{\prime}
\end{array}\right)\left(q_{0}^{\prime}, 0, q_{0}^{\prime}\right)
$$

with

$$
Q^{\prime}=A Q_{s}^{\prime} A^{T}=\left(\begin{array}{ccc}
\frac{1}{2}\left[q_{1}^{\prime}+q_{2}^{\prime}\right]-q_{0}^{\prime 2} & 0 & 0 \\
0 & \frac{1}{2}\left[q_{1}^{\prime}-q_{2}^{\prime}\right] & q_{3}^{\prime} \\
0 & q_{3}^{\prime} & q_{4}^{\prime}
\end{array}\right)
$$

$$
\begin{aligned}
& q_{1}^{\prime}=e^{-2 \Sigma(|\nu-\mu|+|\mu|)} q_{1}+N / 2\left(1-e^{-2 \Sigma(|\nu-\mu|+|\mu|)}\right), \quad q_{2}^{\prime}=e^{-2 \Sigma(|\nu-\mu|+|\mu|)} q_{2} \\
& q_{3}^{\prime}=e^{-\Sigma(|\nu-\mu|+|\mu|)} q_{3} \quad \text { and } \quad q_{4}^{\prime}=q_{4} .
\end{aligned}
$$

Finally,

$$
M_{s}^{\prime}=\left(\begin{array}{ccc}
n_{2}^{\prime} & i n_{3}^{\prime} & n_{1}^{\prime} \\
i n_{4}^{\prime} & 0 & -i n_{4}^{\prime} \\
n_{1}^{\prime} & -i n_{3}^{\prime} & n_{2}^{\prime}
\end{array}\right) \quad \text { and } \quad M^{\prime}=A M_{s}^{\prime} A^{T}=\left(\begin{array}{ccc}
\frac{1}{2}\left[n_{1}^{\prime}+n_{2}^{\prime}\right] & 0 & 0 \\
0 & \frac{1}{2}\left[n_{1}^{\prime}-n_{2}^{\prime}\right] & n_{3}^{\prime} \\
0 & n_{4}^{\prime} & 0
\end{array}\right)
$$

with

$$
\begin{gathered}
n_{1}^{\prime}=e^{-\Sigma(|\nu-\mu|+2|\mu|)} n_{1}, \quad n_{2}^{\prime}=e^{-\Sigma(|\nu-\mu|+2|\mu|)} n_{2}, \\
n_{3}^{\prime}=e^{-\Sigma|\mu|} n_{3} \quad \text { and } \quad n_{4}^{\prime}=e^{-\Sigma(|\nu-\mu|+|\mu|)} n_{4} .
\end{gathered}
$$




\section{Review of quantum metrological sensitivity bounds with OAT}

A fundamental bound to the achievable phase sensitivity is given by the quantum Cramér-Rao bound [47]

$$
\Delta \phi \geq \frac{1}{\sqrt{\mathcal{F}_{Q}\left[\rho_{0}, H\right]}}
$$

this implies

$$
\left(\Delta \phi^{-1}\right)^{2} \leq \mathcal{F}_{Q}\left[\rho_{0}, H\right]
$$

where $\mathcal{F}_{Q}\left[\rho_{0}, H\right]$ denotes the quantum Fisher information of a state $\rho_{0}$ onto which the phase is imprinted by the Hermitian operator $H$. In this case, using the spectral decomposition $\rho_{0}=\sum_{\kappa} p_{\kappa}|\kappa\rangle\langle\kappa|$ with eigenvalues $p_{\kappa} \geq 0$ and associated eigenvectors $\langle\kappa|$, the quantum Fisher information can be expressed as $[7,48]$

$$
\mathcal{F}_{Q}\left[\rho_{0}, H\right]=2 \sum_{\substack{\kappa, \kappa^{\prime} \\ p_{\kappa}+p_{\kappa^{\prime}}>0}} \frac{\left(p_{\kappa}-p_{\kappa^{\prime}}\right)^{2}}{p_{\kappa}+p_{\kappa^{\prime}}}\left|\left\langle\kappa^{\prime}|H| \kappa\right\rangle\right|^{2}
$$

Here we only consider the case of initial states created via OAT, with or without collective dephasing, and unitary rotations with $H=S_{\vec{n}}$ imprinting the phase. Without collective dephasing noise, i.e. $\rho_{0}=T_{\mu}|\mathrm{x}\rangle\langle\mathrm{x}| T_{\mu}^{\dagger}$, it is known that the largest quantum Fisher information is [7]

$$
\max _{\vec{n}} \mathcal{F}_{Q}\left[\rho_{0}, S_{\vec{n}}\right]=\max \left\{N+\frac{N(N-1)}{4}\left(A+\sqrt{A^{2}+B^{2}}\right), N^{2}\left(1-\cos ^{2 N-2}(\mu / 2)\right)-\frac{N(N-1) A}{2}\right\}
$$

with $A=1-\cos ^{N-2}(\mu), B=4 \sin (\mu / 2) \cos ^{N-2}(\mu / 2)$. When including dephasing, in the form of the master equation (9), the density matrix can be expressed in the Dicke basis as

$$
\rho=\sum_{m, m^{\prime}=-N / 2}^{N / 2} e^{-i\left(m^{2}-m^{\prime 2}\right) \mu / 2-\sigma\left(m-m^{\prime}\right)^{2}|\mu| / 4} c_{m}(\theta, \varphi) c_{m^{\prime}}^{*}(\theta, \varphi)|m\rangle\left\langle m^{\prime}\right|
$$

with the coefficients

$$
c_{m}(\theta, \varphi)=\left(\begin{array}{c}
N \\
N / 2+m
\end{array}\right)^{1 / 2} \sin ^{N / 2+m}(\theta / 2) \cos ^{N / 2-m}(\theta / 2) e^{-i(N / 2+m) \varphi}
$$

of the coherent spin state $|\varphi, \theta\rangle$ in the Dicke basis [46]. The quantum Fisher information for the dephased initial state can then be evaluated by numerically diagonalizing $\rho$ based on Eq. 83 and optimizing the right hand side of Eq. 81, with $H=S_{\vec{n}}$, over all directions $\vec{n}$. 

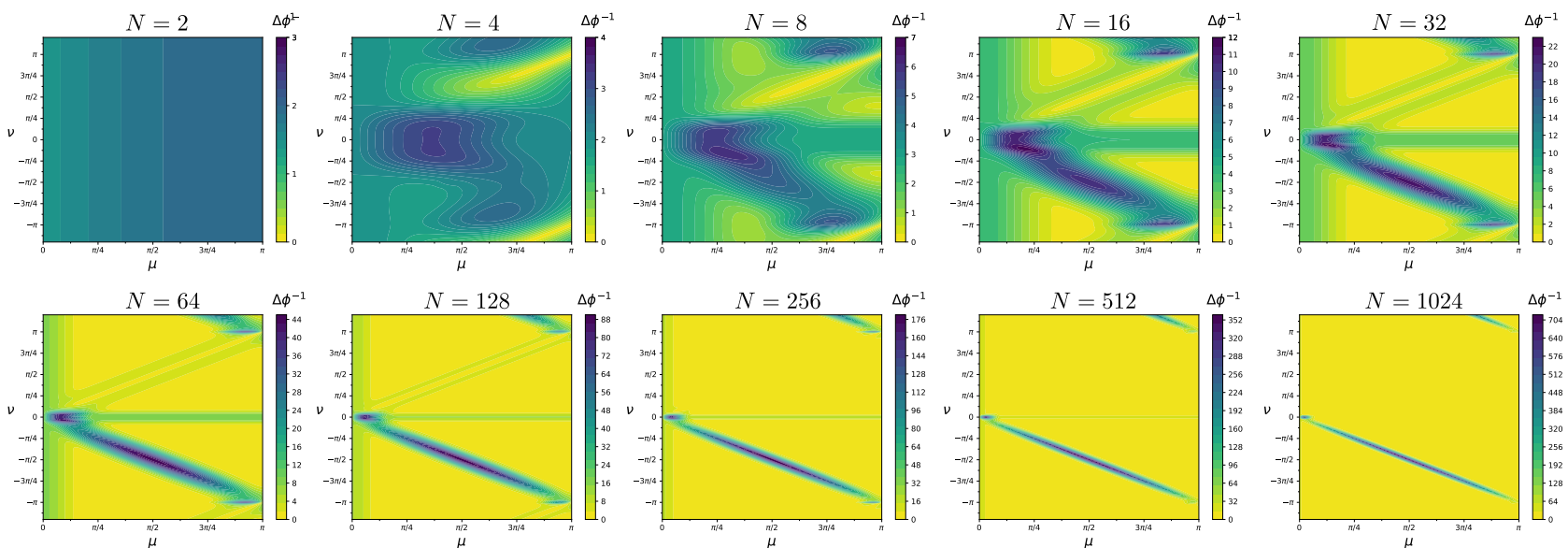

Figure 6: Extended view of the enhancement landscapes, for $N=2-1024$.

(a)
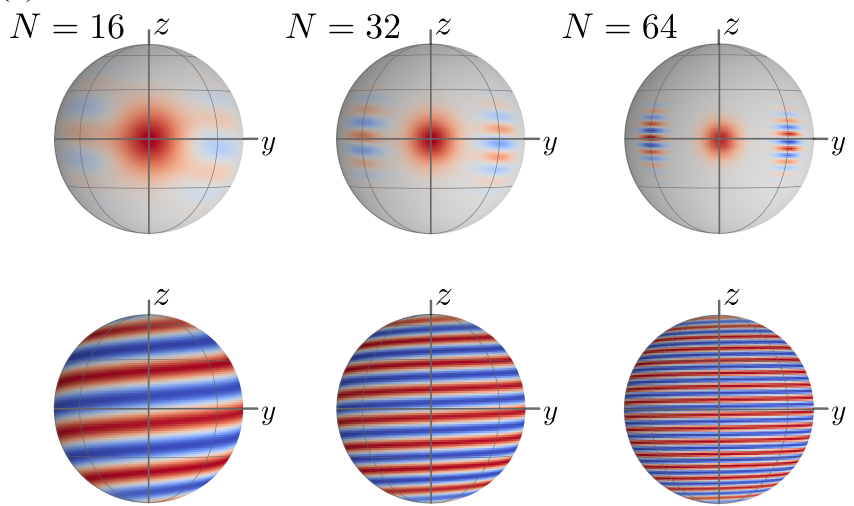

(b)
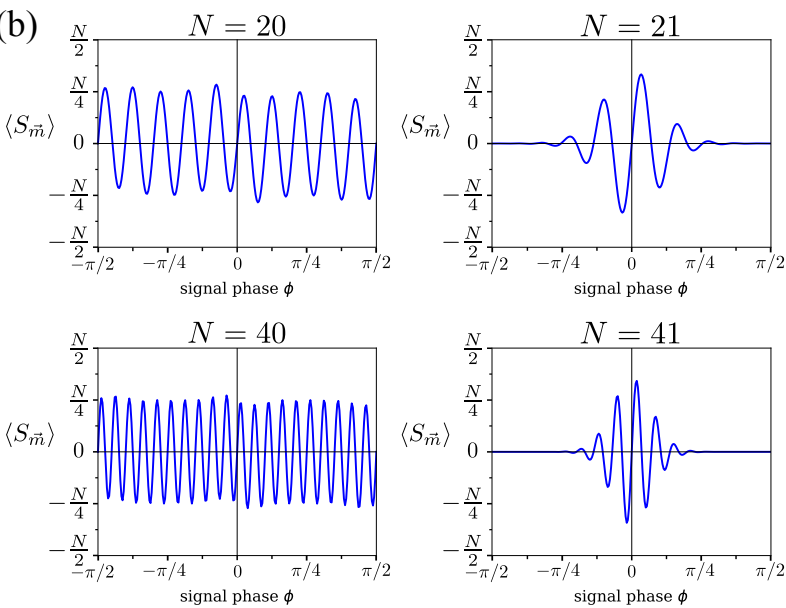

Figure 7: (a) Finer features appearing on the Wigner functions for the state $|\psi\rangle T_{-\mu} R_{y}(\phi) T_{\mu}|\mathrm{x}\rangle$ (upper row) and the measurement operator $T_{\nu}^{\dagger} S_{y} T_{\nu}$ (lower row). (b) OUT signals beyond $\phi \ll 1$. Even particle numbers show an $N$-fold increased oscillation whereas odd particle numbers experience a strong signal for small phases and subsequent attenuation. 